\title{
Overweight and Obesity: Findings from 20 Years of the Tehran Lipid and Glucose Study
}

\author{
Maryam Barzin ${ }^{1}$, Majid Valizadeh ${ }^{1}$, Sara Serahati ${ }^{1}$, Maryam Mahdavi ${ }^{1}$, Fereidoun Azizi ${ }^{2}$ and Farhad \\ Hosseinpanah ${ }^{1, *}$ \\ ${ }^{1}$ Obesity Research Center, Research Institute for Endocrine Sciences, Shahid Beheshti University of Medical Sciences, Tehran, Iran \\ ${ }^{2}$ Endocrine Research Center, Research Institute for Endocrine Sciences, Shahid Beheshti University of Medical Sciences, Tehran, Iran \\ "Corresponding author: MD, Associate Professor of Internal Medicine and Endocrinology, Department of Internal Medicine, School of Medicine, Shahid Beheshti University of \\ Medical Sciences, Tehran, Iran. Tel: +98-2122409309, Fax: +98-2122402463, Email: fhospanah@endocrine.ac.ir
}

Received 2018 September 01; Revised 2018 October 02; Accepted 2018 October 07.

\begin{abstract}
Context: The prevalence of overweight and obesity are increasing worldwide and have frequently been associated with health risks. This review highlighted several studies regarding obesity, outlining contributions of over a span of almost two decades in the Tehran Lipid and Glucose Study (TLGS).

Evidence Acquisition: A systematic review was undertaken to retrieve articles related to all aspects of obesity from the earliest available date up to January 30, 2017.

Results: Prevalence of excess weight, including overweight and obesity were 20.8 and $63.6 \%$ among those aged below and above 20 years, respectively. TLGS found a high incidence of obesity with higher incidence in women among Tehranian adults; the cumulative incidence of obesity was $31.3,38.1$ and $23.4 \%$ for the whole population, women, and men, respectively. In children and adolescents, younger non-obese 7 - 9 years old, compared to 10 - 11 year olds are at greater risk of obesity. Prevalences of abdominal obesity in men and women were $52.8 \%$ and $44.4 \%$ respectively. Similar to generalized obesity, a high incidence of abdominal obesity was observed; the total cumulative incidence of abdominal obesity was 76.0\% (83.6\% for men and 70.9\% for women). Metabolically healthy obese (MHO) and metabolically healthy abdominal obese (MHAO) are two important obesity phenotypes. People with these phenotypes have different risks for cardiovascular disease (CVD), type 2 diabetes (T2DM), and mortality. In the TLGS participants, MHO was found in $2 \%$ and $7.7 \%$ of the whole and obese population, respectively, whereas MHAO phenotype was reported in $12.4 \%$ and $23.5 \%$ of the whole and abdominal obese population. In these unstable conditions, during the long term follow up the metabolic risks developed in nearly half of the individuals. During a 12-year follow-up, incident CVD did not increase in the MHO phenotype compared to metabolically healthy normal weight (MHNW) individuals, but the risk of CVD events had increased in all metabolically unhealthy phenotypes. However in another report, over a 10-year follow-up, MHAO phenotype had an increased risk for CVD in comparison to the reference group, metabolically healthy non-abdominal obese (MHNAO) individuals.

Conclusions: The TLGS studies bridged the significant gap in knowledge regarding prevalence, incidence, trends, morbidities and mortalities for obesity among Iranian population.
\end{abstract}

Keywords: Obesity, Pediatric Obesity, Abdominal Obesity, Morbid Obesity, Metabolically Benign Obesity

\section{Context}

Over the past century, obesity and chronic diseases appear as leading health concerns via shared environmental changes (1). Excess weight is major risks for cardiovascular disease (CVD), type-2 diabetes (T2DM) and mortality (2-4), factors favoring a positive energy balance and weight gain over several decades include increasing per capita highcalorie and palatable food consumption, substitution of occupational physical activities with sitting works with electronic devices, decreasing time spent in leisure-time physical activities and growing time for watching televi- sion, and growing use of medicines that have weight gain as a side effect $(5,6)$.

In this report, we aim to review the 20 year findings of the Tehran Lipid and Glucose Study (TLGS) on obesity and overweight.

\section{Evidence Acquisition}

We searched MEDLINE (via PubMed) for articles published between Jan 1, 2000, and Jan 1, 2018, using the search terms ("obesity" OR “overweight" OR "waist circum- 
ference" OR "body mass index" AND "Tehran Lipid and Glucose Study". All articles with the terms "obesity or overweight" in their title, subject or MeSh were included for initial review. Since studies with nutrition or chronic kidney diseases (CKD) issue as their main topic have been addressed elsewhere, we excluded all articles that have examined the relationship between obesity and nutrition or CKD from this review.

\subsection{Definition of Overweight and Obesity}

For adults, overweight is defined as $25.0 \leq \mathrm{BMI} \leq 29.9$ $\mathrm{kg} / \mathrm{m}^{2}$, and obese is defined as BMI $\geq 30.0 \mathrm{~kg} / \mathrm{m}^{2}$. Obesity is classified by grade as follows: $30 \leq \mathrm{BMI} \leq 34.9$ (class I), $35 \leq$ BMI $\leq 39.9$ (class II), and BMI $\geq 40$ (class III) (7). By WHO definition obesity is defined as BMI-for-age $>2$ SD and overweight is defined as $1 \mathrm{SD}<\mathrm{BMI}-$ for-age $\leq 2 \mathrm{SD}$ in each gender for children (8).

On the other hand, waist circumference (WC) is used to classify excess adiposity which is also associated with increased cardiovascular risk. Iranian National Committee of Obesity defined WC $\geq 90 \mathrm{~cm}$ as at risk for CVD and WC $\geq 95 \mathrm{~cm}$ as high risk for CVD events for both sexes (9).

\section{Results}

\subsection{Youth}

\subsubsection{Prevalence}

In Phase I (1998 to 2001), in children and adolescents aged 3-19 years, the overall prevalence of excess weight (including overweight and obesity) was $20.8 \%$ based on value BMI-for-age $\geq 1$ SD in each gender. The prevalence of excess weight for age group 2 to 5, 6 to 11 years and 12 to 19 years were 13, 19.2 and 23.5\%, respectively. By gender, no significant differences have been reported in prevalence of excess weight.

In addition, obesity prevalence among adolescents with less educated parents was higher than adolescents with high educated parents.

According to data documented between 1998 - 2001 (Phase I), the prevalence of sever obesity which defined as BMI for-age $\geq 3$ SD was $1.2 \%$ for children and adolescents (aged 3 to 19 years). This prevalence rose to 3\% between 2013 - 2016 (Phase V).

\subsubsection{Incidence}

Another analysis of TLGS data, with a median 8.7 years of follow-up showed that among children, aged 7 - 11 years the incidence of obesity was 17\% (CI: 14.7 - 20.3), which in boys was higher than girls [19.5\% (CI: 15.4 - 24.8) vs. $14.5 \%$ (CI:10.9-19.1)]. Compared to non-obese children 10 - 11 years old, 7 - 9 years old were at higher risk of obesity, supported by a cumulative incidence of obesity of $22 \%$ in the younger subgroup, compared with only $10.8 \%$ in older children (Figure 1). The best childhood obesity predictors were overweight, WC more than 95th percentile, hypertension, MetS, and parental obesity (10).

\subsubsection{Trends}

According to TLGS data, between Phases I (1999 - 2001) to Phase IV (2009 to 2011) overall obesity prevalence in youth, aged 3 to 11 years, was increased from $5.5 \%$ to $9.4 \%$. Using GEE (generalized estimating equation) analysis, relative risk of obesity was calculated, comparing each phase to its previous phase: Phase II in reference to Phase I (RR $=1.06, \mathrm{CI}: 1.04-1.08)$, Phase III in reference to Phase II $(\mathrm{RR}=$ 1.01, CI:1.00 -1.03) and Phase IV in reference to Phase III (RR = 0.96, CI: 0.94 - 0.98). Between group difference was significant in all subgroups (age, gender, parental obesity) except for parental education. For children aged below 7 years old in phase I, trend of obesity throughout the study was higher, compared to those $\geq 7$ years of age in this phase.

\subsubsection{Morbidity}

According to TLGS data, the incidence of MetS during 6.6 years follow-up in 6 - 12-year-old children was $10.7 \%$ which was higher in boys compare to girls. Higher BMI and WC were associated with higher incidence and prevalence of MetS and both of these anthropometric indices had the similar power in predicting MetS. Among children aged 6 12-years the cut-off values to predict MetS for BMI were 16.5

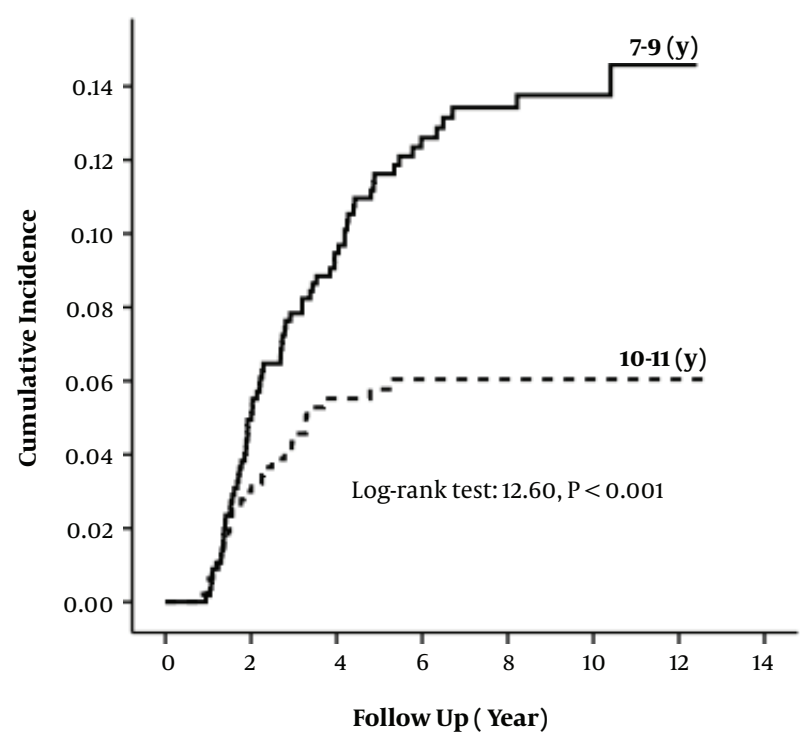

Figure 1. Kaplan-Meier curve for cumulative incidence of obesity (BMI $\geq 30 \mathrm{~kg} / \mathrm{m}^{2}$ ) in different age groups in Tehranian children 
$\mathrm{kg} / \mathrm{m}^{2}$ and $16.3 \mathrm{~kg} / \mathrm{m}^{2}$; for WC were $57.5 \mathrm{~cm}$ and $56.5 \mathrm{~cm}$ for boys and girls, respectively (11).

During a 10.2 year follow-up of 1100 participants with aged 11 - 18 years and without MetS at baseline, the cumulative incidence of MetS in early adulthood for men was 25.5\% and for women was $1.8 \%$. In boys, WC and waist-to-height ratio (WHtR) had the highest risk for the MetS. In age group 11 - 14-year, the results did not change after adjustment for BMI in addition to WC (OR for WC: 2.28 without BMI adjustment vs. 1.98 with BMI adjustment). Therefore, these results showed that beyond BMI, WC predict the risk of MetS. On the other hand, the anthropometric indices did not have significant associations with subsequent MetS risk in girls. Therefore we can conclude that in Tehranian male adolescents abdominal obesity (WHtR and WC) compare to BMI were better predictor for early adulthood MetS (12).

In another study, did not report any association with the risk of MetS in adulthood after adjustment for BMI in adult age, MetS in adolescent age and overweight or obesity. AUC was greater for obesity than MetS(0.619 vs. 0.589). Thus, it seems that independent of adult BMI, adolescent MetS or obesity did not predict early adult MetS (13).

Another analysis was done to explore the association between childhood BMI status (3 - 11 years) and adulthood dysglycemia over a median 9.3 years follow up. The results showed that considering individual and parental factors together, BMI status in early life stages is not associated with the incidence of adult dysglycemia.

\subsection{Adults}

\subsubsection{Prevalence}

In Phase I the overall prevalence of obesity was $23.3 \%$ (29.6 and $14.6 \%$ in women and men, respectively). Overweight was present in $38.3 \%$ of women and $42.4 \%$ of men. In both sexes, the highest rate of obesity was observed in the 50 - 59 year age group. The prevalence of obesity in both sexes increased with age up to 60 years (14).

The prevalence of abdominal obesity in women was greater than men (76.7 vs. $36.5 \%$ ) (15).

\subsubsection{Incidence}

Cumulative incidence of obesity among Tehranian adults in a median 8 year follow up were $31.3,38.1$ and $23.4 \%$ for the whole population, women, and men, respectively. In both sexes, higher risk for development of obesity were higher BMI or WC, MetS and lower educational level at baseline. Men showed the highest incidence rate during their 20s and women during their 40s (Figure 2) (16).

In an investigation with a median 6 years of follow up, total cumulative incidence of abdominal obesity for total population was $76.0 \%$ (83.6\% for men and $70.9 \%$, for women).

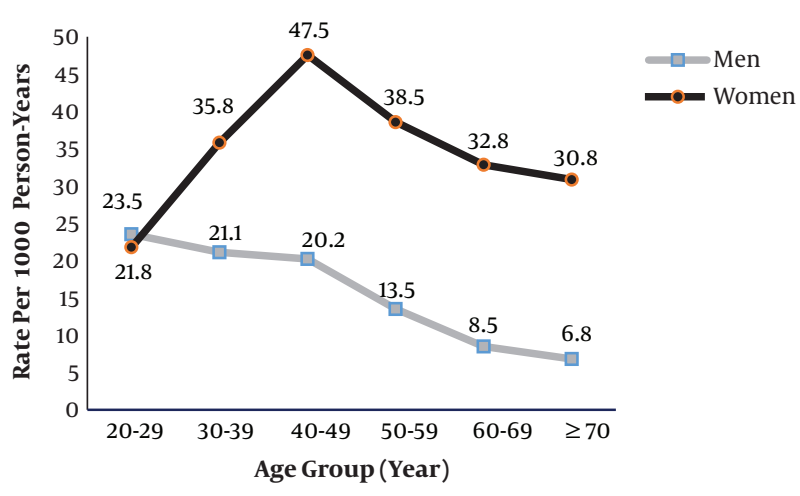

Figure 2. Incidence rates of obesity according to age and sex in Tehranian adults

\subsubsection{Trends}

In both genders, obesity and abdominal had increasing trends across four phases of TLGS in all study subgroups of adults with using generalized estimating equation (GEE) models. The crude prevalence of obesity and abdominal obesity at baseline were 23.1 and $47.9 \%$, respectively. These values increased to 34.1 and $71.1 \%$, at the end of follow-up. Over the whole study period, risks of obesity increased for men and women $(\mathrm{RR}=1.6, \mathrm{CI}: 1.5-1.8$ and $\mathrm{RR}=1.2$, CI:1.2 -1.3, respectively) and abdominal obesity for men and women $(\mathrm{RR}=1.5, \mathrm{CI}: 1.4-1.5$ and $\mathrm{RR}=1.2$, CI: $1.2-1.3$, respectively). Regardless of age, marital status and educational level, these rising trends were observed in all subgroups (Figure 3) (17).

\subsubsection{Obesity Phenotype}

Reports on risk factors for related health outcomes in different obesity phenotypes have yielded contradictory results. A combination of BMI groups and metabolic status developed to different obesity phenotypes; including metabolically obese but normal weight (MONW) and metabolically healthy but obese (MHO). These phenotypes have different risks for incidence of T2DM, CVD and mortality in further (18). Recently, some studies have also used waist circumference (WC) for definition of obesity phenotype.

Prevalence of MHO (based on having less than one component of metabolic syndrome) was $2 \%$ in the total population and 7.7\% in the obese population (19).

Accordingly, the MHAO phenotype (based on abdominal obesity and having less than one component of metabolic syndrome) found in $12 \%$ and $23 \%$ of the total population and the abdominal obese population, respectively (20).

Our investigations showed the MHO phenotype is an unstable condition and half of the individuals developed 


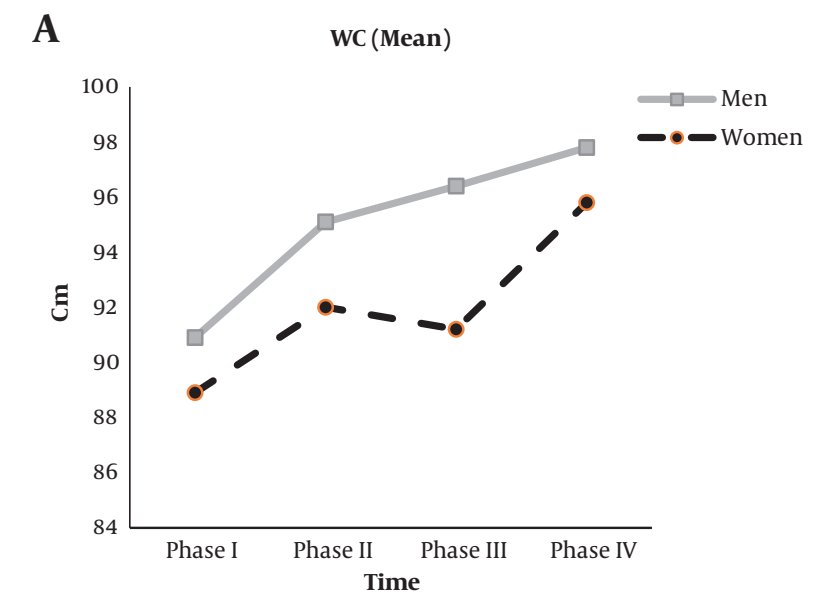

B

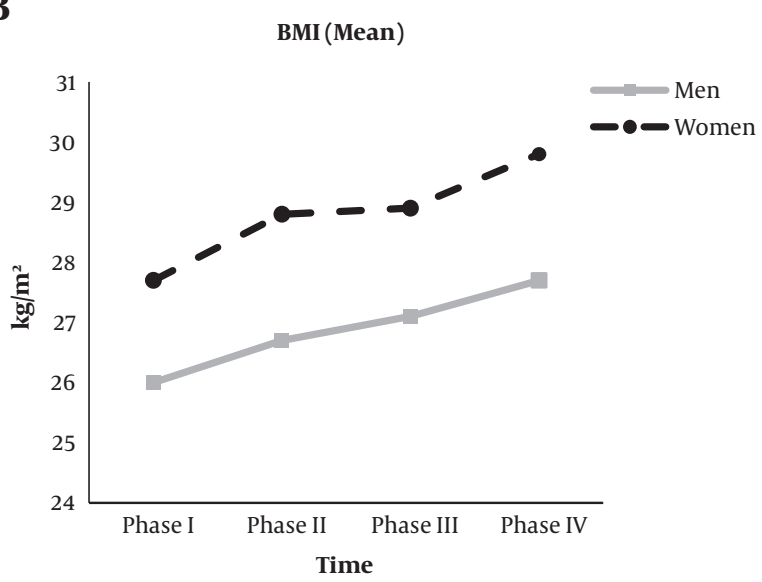

C

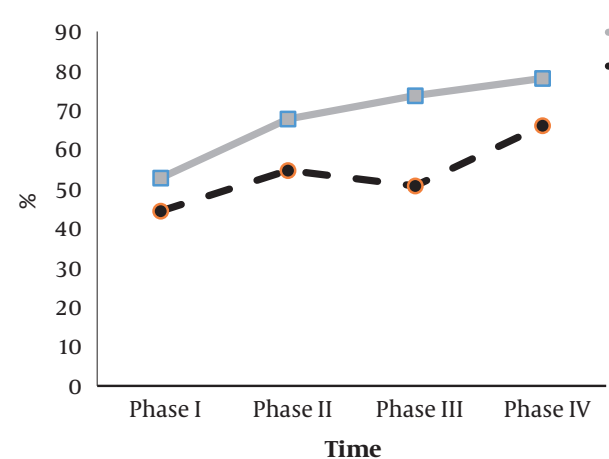

D

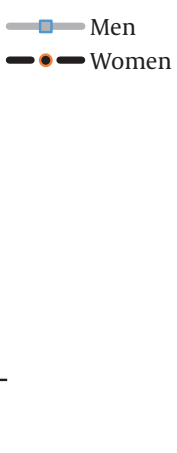

Obesity

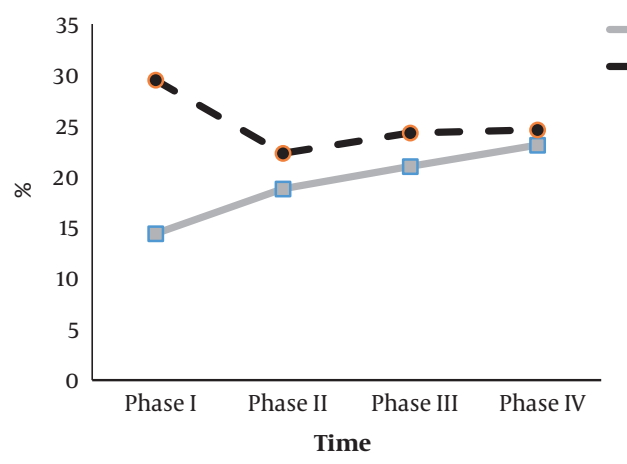

Figure 3. Adult mean levels of (A) waist circumference (WC), (B) body mass index (BMI), (C) prevalence of abdominal obesity, and (D) obesity, across different phases of TLGS

to MetS during 10 years of follow-up (21). Moreover, after 10 years follow-up $43.3 \%$ of MHAO adults lost their metabolic health, of whom 42.1\% developed MetS (22).

During 8.1 years of follow-up, normal-weight subjects with MetS or T2DM compared to overweight and obese subjects without MetS or T2DM had a significantly higher CVD event rate. Also, within the group with MetS or T2DM, a significant association between BMI and CVD events has been observed, whereas no significant association was shown within the group without MetS or T2DM (23).

Moreover, in another report during amount one decade, incident or cardiovascular events did not increase in MHOW and MHO compared to MHNW individuals; however all metabolically unhealthy phenotypes (having more than one metabolic components) showed increased risk of CVD events (Table 1 ) (19).

Our reports during a 10-year follow-up in different abdominal obesity phenotypes (having less than one metabolic component) showed that the risk for CVD was higher in MHAO and MUNAO compared to the reference group; metabolically healthily non-abdominal obese (MHNAO) (20). On the other hand, another study showed that in the MHAO phenotype all-cause mortality risk was not greater than reference group $(\mathrm{HR}=1.3, \mathrm{CI}: 0.9-2.0)(24)$.

Moreover, the association between different obesity phenotypes and incident T2DM was assessed in our cohort. Women with normal weight and MetS compared to healthy normal weight women had ORs for T2DM incidence of 8.8 (CI: 3.7 - 21.2). This values for men were 3.1 (CI: $1.3-7.0)(25)$.

In another report, during 12 years of follow-up the results of multivariate models showed that T2DM risk was increased in all unhealthy abdominal obesity phenotypes (having more than one metabolic component) except the metabolic unhealthy non-abdominal obesity (MUNAO) phenotype in men. Also, MHAO phenotype was associated with incident of T2DM in men ( $\mathrm{HR}=1.5 \mathrm{CI}: 1.0-2.4)$ and in women $(\mathrm{HR}=1.7 \mathrm{CI}: 1.1-2.6)(26)$. 


\begin{tabular}{|c|c|c|c|c|c|c|}
\hline & \multicolumn{3}{|c|}{ Healthy Metabolic Status } & \multicolumn{3}{|c|}{ Unhealthy Metabolic Status } \\
\hline & $\begin{array}{l}\text { Normal Weight } \\
(\mathrm{MHNW})(\mathrm{n}=1218)\end{array}$ & $\begin{array}{c}\text { Overweight } \\
(\text { MHOW })(n=731)\end{array}$ & $\begin{array}{l}\text { Obese (MHO) } \\
\quad(n=147)\end{array}$ & $\begin{array}{l}\text { Normal Weight } \\
(M U N W)(n=920)\end{array}$ & $\begin{array}{c}\text { Overweight } \\
(\text { MUOW })(n=\mathbf{2 4 2 9})\end{array}$ & $\begin{array}{l}\text { Obese (MUHO) } \\
\quad(\mathbf{n}=\mathbf{1 7 2 2})\end{array}$ \\
\hline Unadjusted HR (95\% CI) & 1 & $0.8(0.5-1.3)$ & $1.0(0.5-2.3)$ & $2.8(2.1-3.9)^{\mathrm{d}}$ & $2.9(2.1-3.8)^{\mathrm{d}}$ & $2.5(1.9-3.4)^{\mathrm{d}}$ \\
\hline $\operatorname{Model} 1(95 \% \text { CI })^{b}$ & 1 & $1.2(0.8-1.9)$ & $1.8(0.8-4.0)$ & $1.9(1.4-2.7)^{\mathrm{d}}$ & $2.4(1.8-3.2)^{\mathrm{d}}$ & $2.6(1.9-3.6)^{d}$ \\
\hline Model $2(95 \% \text { CI })^{\text {C }}$ & 1 & $1.2(0.7-2.0)$ & $1.7(0.7-4.4)$ & $1.7(1.2-2.4)^{\mathrm{d}}$ & $2.0(1.5-2.8)^{d}$ & $2.4(1.7-3.5)^{\mathrm{d}}$ \\
\hline
\end{tabular}

Abbreviations: CVD, cardiovascular disease; MHNW, metabolically healthy normal weight; MHO, metabolically healthy obese; MHOW, metabolically healthy over weight; MUHOW: metabolically unhealthy overweight; MUNW, metabolically unhealthy normal weight; MUO: metabolically unhealthy obese.

${ }^{a}$ Metabolic health was defined as one or less of metabolic syndrome components.

${ }^{\mathrm{b}}$ Adjusted for age and sex.

${ }^{c}$ Adjusted for model 1 plus smoking, education level, physical activity, family history of premature coronary artery disease and total cholesterol.

${ }^{\mathrm{d}}$ Compared to reference group (MHNW), $\mathrm{P}<0.001$.

\section{Conclusions}

This review summarizes most of the key findings of the TLGS cohort related to obesity, including prevalence, incidence, trend, and obesity phenotype during 20 years. Overall, similar to other developing countries, we are faced with a high prevalence of obesity and abdominal obesity especially in adulthood. Moreover, the longitudinal nature of TLGS has provides an opportunity to confirm the simultaneous alarming rise of obesity and abdominal obesity, underscoring the urgent need for implement action of preventive strategies aimed of reducing the burden of the problem.

Although a prospective population based study such as TLGS, sheds more light on the various aspects of obesity and its related risk factors, there still remain a certain number of issues which should be resolved in future research. First, considering the limitations of anthropometric indices, we need to gather data regarding body compositions. Second, in order to assess the impact of socioeconomic status on different aspects of obesity, more information is needed to determine this variable. Third, to obtain a bigger picture of the problem at a national level, we can merge our findings with the results of other cohorts in Iran. Fourth, research on genetic causes of all types of obesity and related comorbidities must be conducted in the future. Also, in the context of assessing the impact of obesity during adolescence on cardiovascular outcomes in early adulthood, providing some surrogate endpoint such as intimal media thickness can overcome the problem of low event rate in this age category.

\section{References}

1. Ng M, Fleming T, Robinson M, Thomson B, Graetz N, Margono C, et al. Global, regional, and national prevalence of overweight and obesity in children and adults during 1980-2013: A systematic analysis for the Global Burden of Disease Study 2013. Lancet. 2014;384(9945):766-81. doi: 10.1016/S0140-6736(14)60460-8. [PubMed: 24880830]. [PubMed Central: PMC4624264].
2. Fan J, Song Y, Chen Y, Hui R, Zhang W. Combined effect of obesity and cardio-metabolic abnormality on the risk of cardiovascular disease: A meta-analysis of prospective cohort studies. Int J Cardiol. 2013;168(5):4761-8. doi: 10.1016/j.ijcard.2013.07.230. [PubMed: 23972953].

3. Hossain P, Kawar B, El Nahas M. Obesity and diabetes in the developing world-a growing challenge. N Engl J Med. 2007;356(3):213-5. doi: 10.1056/NEJMp068177. [PubMed: 17229948].

4. Afshin A, Forouzanfar MH, Reitsma MB, Sur P, Estep K; GBD 2015 Obesity Collaborators, et al. Health effects of overweight and obesity in 195 countries over 25 years. N Engl J Med. 2017;377(1):13-27. doi: 10.1056/NEJMoa1614362. [PubMed: 28604169]. [PubMed Central: PMC5477817].

5. Biddle SJH, Garcia Bengoechea E, Pedisic Z, Bennie J, Vergeer I, Wiesner G. Screen time, other sedentary behaviours, and obesity risk in adults: A review of reviews. Curr Obes Rep. 2017;6(2):134-47. doi: 10.1007/s13679-017-0256-9. [PubMed: 28421472].

6. Bornhorst C, Wijnhoven TM, Kunesova M, Yngve A, Rito AI, Lissner L, et al. WHO European Childhood Obesity Surveillance Initiative: Associations between sleep duration, screen time and food consumption frequencies. BMC Public Health. 2015;15:442. doi: 10.1186/s12889-015-1793-3. [PubMed: 25924872]. [PubMed Central: PMC4440513].

7. [No authors listed]. NIH conference. Gastrointestinal surgery for severe obesity. Consensus Development Conference Panel. Ann Intern Med.1991;115(12):956-61. [PubMed: 1952493].

8. World Health Organization. BMI-for-age ( 5 - 19 years). 2006.

9. Azizi F, Khalili D, Aghajani H, Esteghamati A, Hosseinpanah F, Delavari A, et al. Appropriate waist circumference cut-off points among Iranian adults: The first report of the Iranian National Committee of Obesity. Arch Iran Med. 2010;13(3):243-4. [PubMed: 20433230].

10. Barzin M, Aryannezhad S, Serahati S, Beikyazdi A, Azizi F, Valizadeh M, et al. Incidence of obesity and its predictors in children and adolescents in 10 years of follow up: Tehran Lipid And Glucose Study (TLGS). BMC Pediatr. 2018;18(1):245. doi: 10.1186/s12887-018-1224-6. [PubMed: 30045707]. [PubMed Central: PMC6060527].

11. Barzin M, Hosseinpanah F, Fekri S, Azizi F. Predictive value of body mass index and waist circumference for metabolic syndrome in 6-12-year-olds. Acta Paediatr. 2011;100(5):722-7. doi: 10.1111/j.16512227.2011.02162.x. [PubMed: 21244485].

12. Barzin M, Asghari G, Hosseinpanah F, Mirmiran P, Azizi F. The association of anthropometric indices in adolescence with the occurrence of the metabolic syndrome in early adulthood: Tehran Lipid and Glucose Study (TLGS). Pediatr Obes. 2013;8(3):170-7. doi: 10.1111/j.20476310.2012.00102.x. [PubMed: 23042576].

13. Hosseinpanah F, Asghari G, Barzin M, Ghareh S, Azizi F. Adolescence metabolic syndrome or adiposity and early adult 
metabolic syndrome. J Pediatr. 2013;163(6):1663-1669 e1. doi: 10.1016/j.jpeds.2013.07.032. [PubMed: 24011762].

14. Azizi F, Esmaillzadeh A, Mirmiran FP. Obesity and cardiovascular disease risk factors in Tehran adults: A population-based study. East Mediterr Health J. 2004;10(6):887-97. [PubMed: 16335777].

15. Hosseinpanah F, Barzin M, Eskandary PS, Mirmiran P, Azizi F. Trends of obesity and abdominal obesity in Tehranian adults: A cohort study. BMC Public Health. 2009;9:426. doi: 10.1186/1471-2458-9-426. [PubMed: 19930614]. [PubMed Central: PMC2801677].

16. Barzin M, Hosseinpanah F, Motamedi MA, Shapoori P, Arian P, Daneshpour MA, et al. Bariatric surgery for morbid obesity: Tehran Obesity Treatment Study (TOTS) rationale and study design. JMIR Res Protoc. 2016;5(1). e8. doi: 10.2196/resprot.5214. [PubMed: 26792554]. [PubMed Central: PMC4740496].

17. Barzin M, Keihani S, Hosseinpanah F, Serahati S, Ghareh S, Azizi F. Rising trends of obesity and abdominal obesity in 10 years of follow-up among Tehranian adults: Tehran Lipid and Glucose Study (TLGS). Public Health Nutr. 2015;18(16):2981-9. doi: 10.1017/S1368980015000269. [PubMed: 25711365].

18. Ruderman N, Chisholm D, Pi-Sunyer X, Schneider S. The metabolically obese, normal-weight individual revisited. Diabetes. 1998;47(5):699713. [PubMed: 9588440].

19. Mirzaei B, Abdi H, Serahati S, Barzin M, Niroomand M, Azizi F, et al. Cardiovascular risk in different obesity phenotypes over a decade followup: Tehran Lipid and Glucose Study. Atherosclerosis. 2017;258:65-71. doi: 10.1016/j.atherosclerosis.2017.02.002. [PubMed: 28213199].

20. Keihani S, Hosseinpanah F, Barzin M, Serahati S, Doustmohamadian S, Azizi F. Abdominal obesity phenotypes and risk of cardiovascular disease in a decade of follow-up: The Tehran
Lipid and Glucose Study. Atherosclerosis. 2015;238(2):256-63. doi: 10.1016/j.atherosclerosis.2014.12.008. [PubMed: 25540856].

21. Hosseinpanah F, Nazeri P, Ghareh S, Tohidi M, Azizi F. Predictors of the incident metabolic syndrome in healthy obese subjects: A decade of follow-up from the Tehran Lipid and Glucose Study. Eur J Clin Nutr. 2014;68(3):295-9. doi: 10.1038/ejcn.2013.142. [PubMed: 23963276].

22. Eshtiaghi R, Keihani S, Hosseinpanah F, Barzin M, Azizi F. Natural course of metabolically healthy abdominal obese adults after 10 years of follow-up: The Tehran Lipid and Glucose Study. Int J Obes (Lond). 2015;39(3):514-9. doi: 10.1038/ijo.2014.176. [PubMed: 25287753].

23. Hosseinpanah F, Barzin M, Sheikholeslami F, Azizi F. Effect of different obesity phenotypes on cardiovascular events in Tehran Lipid and Glucose Study (TLGS). Am J Cardiol. 2011;107(3):412-6. doi: 10.1016/j.amjcard.2010.09.034. [PubMed: 21257007].

24. Doustmohamadian S, Serahati S, Barzin M, Keihani S, Azizi F, Hosseinpanah F. Risk of all-cause mortality in abdominal obesity phenotypes: Tehran Lipid and Glucose Study. Nutr Metab Cardiovasc Dis. 2017;27(3):241-8. doi: 10.1016/j.numecd.2016.11.123. [PubMed: 28139376].

25. Hadaegh F, Bozorgmanesh M, Safarkhani M, Khalili D, Azizi F. Predictability of body mass index for diabetes: Affected by the presence of metabolic syndrome? BMC Public Health. 2011;11:383. doi: 10.1186/1471-2458-11-383. [PubMed: 21609497]. [PubMed Central: PMC3119166].

26. Salehinia F, Abdi H, Hadaegh F, Serahati S, Valizadeh M, Azizi F, et al Abdominal obesity phenotypes and incident diabetes over 12 years of follow-up: The Tehran Lipid and Glucose Study. Diabetes Res Clin Pract. 2018;144:17-24. doi: 10.1016/j.diabres.2018.07.021. [PubMed: 30036611] 